\title{
PREVALENCIA DE MANIFESTACIONES ORALES EN PACIENTES HIPERTENSOS CON MEDICACIÓN CONTINUA, HOSPITAL JACOBO Y MARÍA ELENA RATINOFF, AÑO 2014
}

PREVALENCE OF ORAL MANIFESTATIONS IN PATIENTS HYPERTENSIVE WITH CONTINUOUS MEDICATION, JACOBO AND MARÍA ELENA RATINOFF HOSPITAL, YEAR 2014

PREVALÊNCIA DE MANIFESTAÇ̃̃ES ORAIS EM PACIENTES HIPERTENSO COM MEDICAÇÃO CONTÍNUA, HOSPITAL JACOBO E MARÍA ELENA RATINOFF, ANO DE 2014

\section{GEOCONDA LUZARDO JURADO, NICOLE RAAD BASSIL}

Universidad Católica de Santiago de Guayaquil; Guayaquil, Ecuador.

Resumen

Problema: los medicamentos antihipertensivos pueden causar afecciones orales tales como xerostomía, disgeusia e hiperplasia gingival. Objetivo: determinar la prevalencia de manifestaciones orales en pacientes hipertensos con medicación continua. Metodología: estudio de tipo transversal en el que se incluyeron 113 pacientes hipertensos que asistieron a la consulta del hospital del día Jacobo y Maria Elena Ratinoff. La historia clínica, registra hiposalivación y disgeusia, gingivitis. Resultados: 10 pacientes pertenecieron al grupo entre 40-49 años, 29 pacientes al grupo de 50 - 59 años, 43 pacientes al de 60-69 años, 25 pacientes entre los $70-79$ años y 6 pacientes entre los 80 -89 años. El 80.53\% de los pacientes presentaron manifestaciones orales. La manifestación más común fue disgeusia con un $60 \%$, seguido de hiposalivación con un $58 \%$ y agrandamiento gingival siendo la menos prevalente con un $12 \%$. Además la hiposalivación y disgeusia predominaron en mujeres con un $69 \%$ y $67 \%$ respectivamente. Sin embargo el agrandamiento gingival fue más abundante en hombres con un 14\%. Conclusión: se encontró un alto porcentaje de pacientes hipertensos con presencia de manifestaciones orales relacionadas a los medicamentos.

PALABRAS CLAVE: hipertensión, medicamentos antihipertensivos, xerostomía, disgeusia, hiperplasia gingival.

Abstract

Problem: the intake of antihypertensive drugs can cause oral manifestations like dry mouth, dysgeusia and gingival hyperplasia. Objective: the aim of this study was to determine the prevalence of oral manifestations in hypertensive patients with medication. Materials and methods: it was an opportunity study in which 113 hypertensive patients were involved. All the patients attended Hospital del día Jacobo y María Elena Ratinoff. Patients were interviewed through a clinical history, dysgeusia and hyposalivation records were taken, as well as an intraoral examination. Results: 10 patients belonged to the group of 40-49 years, 29 patients to the group of 50-59 years, 43 patients were between $60-69$ years, 25 patients had between 70-79 years and 6 patients were from $80-89$ years. We noticed that $80.53 \%$ of patients had oral manifestations. The most common manifestation was dysgeusia with $60 \%$, followed by hyposalivation with $58 \%$ and gingival enlargement being less prevalent with $12 \%$. Also we found that dysgeusia and hyposalivation were more prevalent in women with $69 \%$ and $67 \%$ respectively. However, gingival overgrowth was more prevalent in men with $14 \%$. Conclusion: a high percentage of hypertensive patients were found to have oral manifestations due to drug intake

Keywords: hypertension, antihypertensive agents, xerostomia, dysgeusia, gingival hyperplasia.

Resumo

Problema: os medicamentos anti-hipertensivos podem causar condições bucais como xerostomia, disgeusia e hiperplasia gengival. Objetivo: determinar a prevalência de manifestações orais em pacientes hipertensos com medicação contínua. Materiais e métodos: estudo transversal que incluiu 113 pacientes hipertensos que compareceram à consulta hospitalar de Jacobo e Maria Elena Ratinoff. A história médica registra hipossalivação e disgeusia, gengivite. Resultados: 10 pacientes pertenceram ao grupo entre 40-49 anos, 29 pacientes ao grupo de 50-59 anos, 43 pacientes ao grupo de 60-69 anos, 25 pacientes entre 70-79 anos e 6 pacientes entre 80-89 anos. 80,53\% dos pacientes apresentaram manifestações orais. A manifestação mais comum foi disgeusia com $60 \%$, seguida de hipossalivação com $58 \%$ e aumento gengival, sendo a menos prevalente com $12 \%$. Além disso, a hipossalivação e a disgeusia predominaram em mulheres com 69\% e 67\%, respectivamente. № entanto, 0 aumento gengival foi mais abundante em homens com 14\%. Conclusão: foi encontrado um alto percentual de hipertensos com presença de manifestações orais relacionadas a medicamentos. PALABRAS-CHAVE: hipertensão, anti-hipertensivos, xerostomia, disgeusia, hiperplasia gingival. 
INTRODUCCIÓN

La terapia medicamentosa en pacientes hipertensos es recomendada con una presión arterial mayor o igual a 140/90 $\mathrm{mmHg}$. Es importante que un paciente hipertenso se encuentre controlado siempre, ya que esto confiere una reducción de hasta el 40\% del riesgo de sufrir un accidente cerebrovasculary de un $16 \%$ de sufrir enfermedades cardíacas en los 5 años siguientes al tratamiento. Además reduce hasta un $50 \%$ el riesgo de insuficiencia cardíaca. ${ }^{1}$ Actualmente no se ha establecido en nuestro medio la relación entre el uso de fármacos anti-hipertensivos con la patogenia de las manifestaciones orales descritas

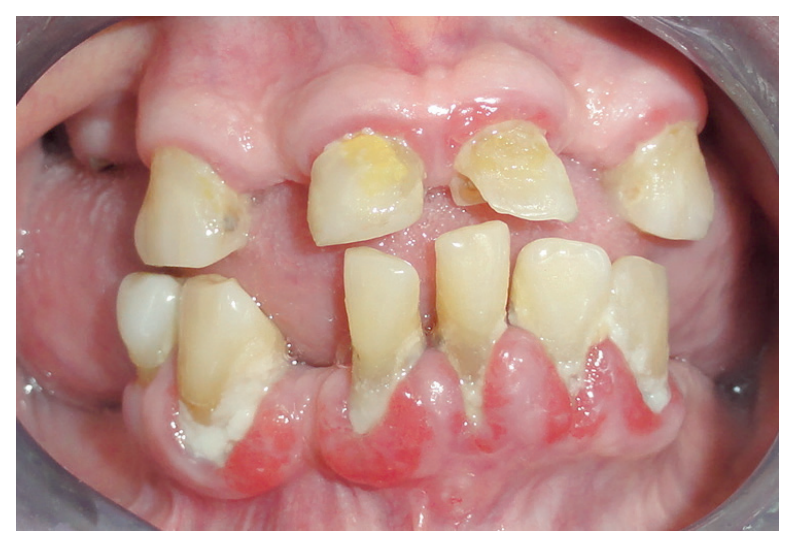

Figura 1. Agrandamiento gingival en paciente hipertenso tomando Amlodipina.

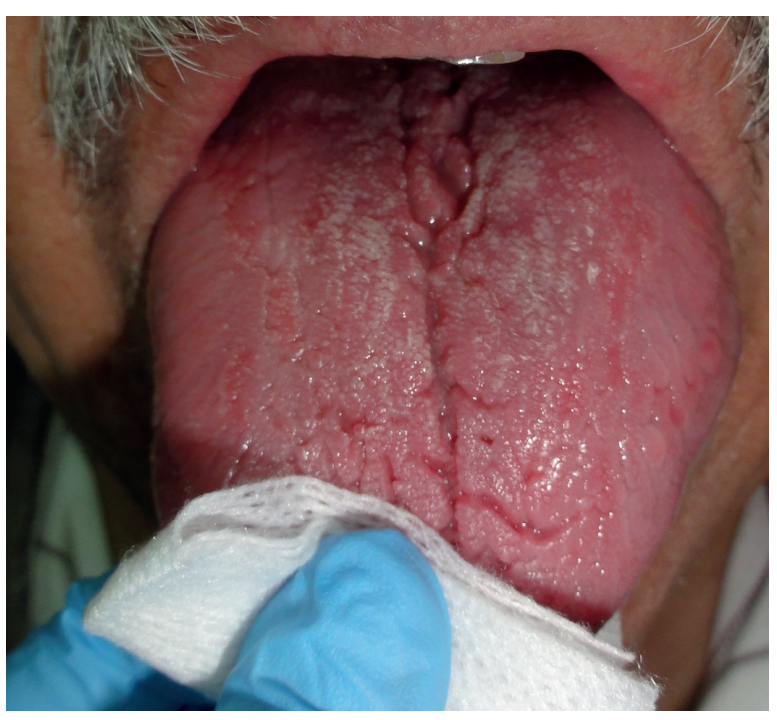

Figura 2. Lengua fisurada a causa de hiposalivación en paciente hipertenso.

Los fármacos utilizados pertenecen al grupo de los diuréticos, beta bloqueantes, simpaticolíticos, alfa antagonistas y los antagonistas de los canales de calcio. ${ }^{1}$ y su uso puede causar manifestaciones orales tales como xerostomía, disgeusia, hiperplasia gingival, reacciones liquenoides, entre otras como efecto secundario.
Hasta la actualidad no se conoce de manera clara la etiología o patogénesis de la hiperplasia gingival inducida por fármacos. Ciertas teorías se han enfocado en el efecto directo que tiene la droga o sus metabolitos sobre los fibroblastos gingivales. Sin embargo ninguna de las teorías explica porque algunos individuos son afectados y otros no, por lo que se considera que intervienen, además, otros factores como predisposición genética, variables farmacocinéticas y factores de inflamación. ${ }^{5}$ Debido a esto es importante conocer los medicamentos son los causantes de tales manifestaciones así como la frecuencia con la cual éstas aparecen.

Con estos antecedentes, y debido a que un $28.7 \%$ de la población ecuatoriana fue diagnosticada con hipertensión en el año 2009, es importante conocer las posibles lesiones orales que se pueden presentar y la prevalencia de éstas en los pacientes hipertensos medicados. Este estudio nos ayudaráa comprender el estado de salud oral de los pacientes hipertensos con medicación continua, para asídar a conocer los cuidados y precauciones que deben tener en cuenta, tanto los pacientes, como médicos y odontólogos.

\section{METODOLOGÍA}

Se realizó un estudio descriptivo, cuyo diseño es transversal. El universo estuvo conformado por pacientes hipertensos que asistieron al hospital del día Jacobo y María Elena Ratinoff en la ciudad de Guayaquil, febrero de 2014.

La población de estudio estuvo conformada por un total de 113 pacientes hipertensos diagnosticados, mayores de edad y que estaban tomando medicación para controlar la hipertensión. No se incluyeron aquellos pacientes que no firmaron el consentimiento informado y se negaron a participar en el presente estudio.

Además, fueron excluidos pacientes con enfermedades sistémicas o graves tales como diabetes, VIH y lupus eritematoso sistémico; pacientes que tomaban otro tipo de medicamentos que pudieran interferir en el estudio como anticonvulsivos o inmunosupresores y pacientes embarazadas.

Se excluyeron 3 pacientes quienes llevaban tomando menos de 3 meses la medicación antihipertensiva. El estudio se realizó a partir de las 8 de la mañana revisando el expediente clínico de los pacientes que asistieron a la consulta cardiológica durante 3 semanas del mes de febrero del año 2014. (Figura 3). 


\section{FIGURA 3. HOJA DE REGISTRO Y ENCUESTA REALIZADA A PACIENTES DURANTEEL ESTUDIO}

\begin{tabular}{|c|c|c|}
\hline \multirow{2}{*}{$\begin{array}{c}\text { "Prevalencia de manifestaciones orales en pacien- } \\
\text { tes hipertensos medicados" }\end{array}$} & MEDICAMENTOS & \multirow[t]{2}{*}{ 2.4 Registro de Hiposalivación } \\
\hline & IECA: & \\
\hline \multirow{3}{*}{ Universidad Católica de Santiago de Guayaquil } & Captopril & \multirow{4}{*}{$\begin{array}{l}\text { 1. ¿Siente usualmente la boca seca? } \\
\text { 2. ¿Siente la boca seca mientras come? } \\
\text { 3. ¿Tiene dificultad para tragar alimentos secos? } \\
\text { 4. ¿Necesita dela ayuda de líquidos para tragar los } \\
\text { alimentos? }\end{array}$} \\
\hline & Enalapril & \\
\hline & Lisinopril & \\
\hline Nicole Raad Bassil & Landesartan & \\
\hline Historia Clínica N: & Calcio antagonistas: & \multirow{2}{*}{$\begin{array}{l}\text { 5. ¿Siente poca cantidad de saliva en su boca o no } \\
\text { lo ha notado? }\end{array}$} \\
\hline & Verapamilo & \\
\hline 1. Datos generales & Diuréticos: & \\
\hline \multirow[t]{3}{*}{ Num. de cédula__________ Sexo____ } & Hydroclorotiazidas & \\
\hline & \begin{tabular}{|l|} 
Clortalidona \\
Furosemida
\end{tabular} & \multirow{4}{*}{$\begin{array}{l}\text { gusto? } \\
\text { 4. Al Al comemer iSientela comida sin sabor? } \\
\text { 5. ¿Sienteinapetencia? } \\
\text { 3. Examen clínico }\end{array}$} \\
\hline & Bloqueadores $\beta$ adrenérgicos & \\
\hline Ocupación:_______Estado civil:___________ & Propanolol & \\
\hline \multirow{7}{*}{$\begin{array}{l}\text { 2. Anamnesis } \\
\text { 2.1 ¿Hace cuanto fue diagnosticado como hiper- } \\
\text { tenso? } \\
\text { 2.2 ¿Desde cuando toma la medicación actual? } \\
\text { 2.3¿Qué medicamentos toma? }\end{array}$} & ау $\beta$ bloqueantes adrenérgicos: & \\
\hline & Metildopa & 3. 1 Localización de la lesión \\
\hline & Vasodilatadores & 3.2 Diagnóstico de la lesión \\
\hline & Hydralazina & Xerostomía / Hiposalivación \\
\hline & \begin{tabular}{|l|} 
Minoxidil| \\
Inhibidores de la renina:
\end{tabular} & Disgeusia / Alteración del gusto \\
\hline & Aliskireno & Hiperplasia gingival \\
\hline & OTROS & \begin{tabular}{|l|} 
Reacciones liquenoides \\
\end{tabular} \\
\hline
\end{tabular}

Usando todas las medidas de bioseguridad se procedió a la examinación intraoral de cada paciente, específicamente de los tejidos blandos, usando 2 espejos intraorales, una pinza algodonera, torundas y gasas. En caso de encontrar una patología o manifestación producto de la ingesta de un medicamento antihipertensivo, este hallazgo fue registrado en la historia clínica del estudio. Luego se procedió a tomar fotografías de las manifestaciones encontradas.

Una vez terminado de recopilar toda la información, se tabuló y se elaboró una tabla en una hoja de cálculo en el programa Microsoft Excel. Se procesó la información en la hoja de cálculo, el análisis estadístico fue básicamente descriptivo con medidas de tendencia céntrlca como promedio, porcentajes y además se hizo un análisis comparativo mediante Chi2.

\section{RESULTADOS}

Del total de 113 pacientes hipertensos que asistieron a la consulta cardiológica del hospital del día Jacobo y María Elena Ratinoff se encontró que 10 tenían entre 40 - 49 años $(8.85 \%), 29$ pacientes entre 50 - 59 años $(25.66 \%), 43$ pacientes entre $60-69$ años (38.05\%), 25 pacientes entre los $70-79$ años $(22.12 \%$ ) y 6 pacientes entre los $80-89$ años (5.31\%). 85 fueron mujeres $(75.22 \%$ ) y 28 hombres (24.78\%). (Tabla 1 )

De los medicamentos antihipertensivos, el que se detectó con mayor prevalencia fue el losartán con un $86 \%$, seguido de atenolol con un $35 \%$, amlodipina con $22 \%$ y enalapril con $20 \%$. (Tabla 1 )
TABLA2. DISTRIBUCIÓN DE LA MUESTRA DEACUERDO A LA EDAD, GÉNERO EINGESTA DE MEDICAMENTOS

\begin{tabular}{lll}
\hline EDAD & Frecuencia & Porcentaje \\
\hline Edad & 10 & $8.85 \%$ \\
\hline $50-49$ & 29 & $25.66 \%$ \\
\hline $60-59$ & 43 & $38.05 \%$ \\
\hline $70-79$ & 25 & $22.12 \%$ \\
\hline $80-89$ & 6 & $5.31 \%$ \\
\hline Total & 113 & $100.00 \%$ \\
\hline GÉNERO & & \\
\hline Género & Frecuencia & Porcentaje \\
\hline Femenino & 85 & $75.22 \%$ \\
\hline Masculino & 28 & $24.78 \%$ \\
\hline Total & 113 & $100.00 \%$ \\
\hline INGESTA DE MEDICAMENT0 & \\
\hline Medicamentos & Frecuencia & Porcentaje \\
\hline Enalapril & 23 & $20 \%$ \\
\hline Losartán & 97 & $86 \%$ \\
\hline Amlodipina & 25 & $22 \%$ \\
\hline Nifedipina & 2 & $2 \%$ \\
\hline Hydroclorotiazidas & 2 & $2 \%$ \\
\hline Clortalidona & 1 & $1 \%$ \\
\hline Furosemida & 2 & $2 \%$ \\
\hline Atenolol & 39 & $35 \%$ \\
\hline & & \\
\hline
\end{tabular}

Se determinó que 91 pacientes (80.53\%) presentaron algún tipo de manifestación oral y 22 (19.47\%) no presentaron. (Figura 3) La manifestación oral que se encontró con mayor prevalencia fue disgeusia con un $60 \%$, seguido de hiposalivación con un $58 \%$ y agrandamiento gingival siendo la menos prevalente con un $12 \%$. 


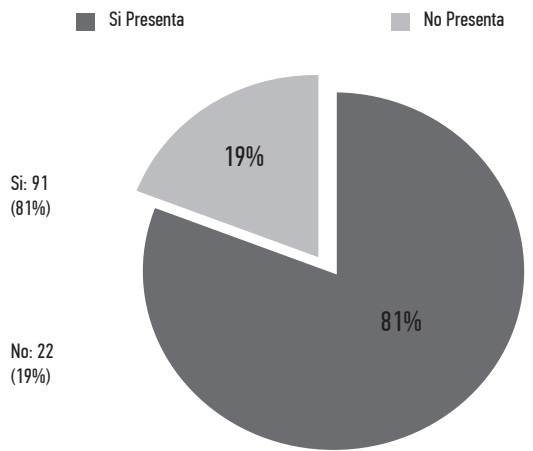

Figura 3. Prevalencia de manifestaciones orales en pacientes hipertensos.

Durante el registro de hiposalivación, del total de pacientes, 67 (59.29\%) manifestaron que usualmente sentían la boca seca y 39 (34.51\%) manifestaron que sentían poca cantidad de saliva en su boca. (Figura 2).

En el registro de disgeusia, 68 (60.18\%) pacientes manifestaron que sentían usualmente un mal sabor en la boca y 66 (58.41\%), que sentían un sabor metálico en la boca. De los pacientes que estaban tomando enalapril se encontró que la manifestación de mayor prevalencia fue hiposalivación con un $65 \%$, seguido de disgeusia con un $60 \%$ y por último agrandamiento gingival con un $13 \%$. Quienes estaban tomando amlodipino, la manifestación de mayor prevalencia fue disgeusia con un $64 \%$, seguido de hiposalivación con un $60 \%$ y por ultimo agrandamiento gingival con un $20 \%$. De los pacientes que estaban tomando losartán, la manifestación de mayor prevalencia fue hiposalivación con un $58 \%$, seguido de disgeusia con un $58 \%$ y agrandamiento gingival con un $12 \%$. De quienes toman atenolol la manifestación de mayor prevalencia fue disgeusia con un $64 \%$, seguido de hiposalivación con un 56\% y agrandamiento gingival con un $20 \%$. (Tabla 3 ).

TABLA 3. RELACIÓN DELA INGESTA DEMEDICAMENTOS ANTIHIPERTENSIVOS YLA PRESENCIA DE MANIFESTACIONES ORALES

\begin{tabular}{|c|c|c|}
\hline \multicolumn{3}{|l|}{ ENALAPRIL } \\
\hline & Si & No \\
\hline Hiposalivación & $15(65 \%)$ & $8(34 \%)$ \\
\hline Disgeusia & $14(60 \%)$ & $9(39 \%)$ \\
\hline Agrandamiento gingival & $3(13 \%)$ & $20(86 \%)$ \\
\hline \multicolumn{3}{|l|}{ LOSARTAN } \\
\hline & Si & No \\
\hline Hiposalivación & $57 \quad(58 \%)$ & $40(41 \%)$ \\
\hline Disguesia & $56 \quad(57 \%)$ & $41(42 \%)$ \\
\hline Agrandamiento gingival & $12(12 \%)$ & $85(87 \%)$ \\
\hline \multicolumn{3}{|l|}{ AMLODIPINA } \\
\hline & Si & No \\
\hline Hiposalivación & $15(60 \%)$ & $10(40 \%)$ \\
\hline Disguesia & $16 \quad(64 \%)$ & $9(36 \%)$ \\
\hline Agrandamiento gingival & $5(20 \%)$ & $20(80 \%)$ \\
\hline \multicolumn{3}{|l|}{ ATENELOL } \\
\hline & $\mathrm{Si}$ & No \\
\hline Hiposalivación & $22(56 \%)$ & $17(43 \%)$ \\
\hline Disguesia & $25 \quad(64 \%)$ & $14(35 \%)$ \\
\hline Agrandamiento gingival & $8(20 \%)$ & $31(79 \%)$ \\
\hline
\end{tabular}

Tanto la hiposalivación, como disgeusia y agrandamiento gingival se presentaron en pacientes que llevaban menos de un año de ingesta del medicamento. La disgeusia e hiperplasia gingival fueron más frecuentes en pacientes que llevaban tomando más de tres años la medicación antihipertensiva con $69 \%$ y $21 \%$ respectivamente . Sin embargo la hiposalivación fue más prevalente en pacientes que llevaban tomando menos de un año el medicamento con un $63 \%$.

Del total de la muestra, la hiposalivación y la disgeusia fueron más prevalentes en mujeres con un $69 \%$ y $67 \%$ respectivamente; sin embargo en cuanto al agrandamiento gingival se encontró una mayor prevalencia en hombres con un $14 \%$. (Tabla 4).

TABLA 4. RELACIÓN ENTRE EL GÉNERO Y LA PREVALENCIA DE MANIFESTACIONES ORALES

\begin{tabular}{l|r|r|r|r}
\hline GÉNERO & \multicolumn{3}{|c}{ MASCULINO } & FEMENINO \\
& SI & NO & SI & NO \\
\hline Hiposalivación & $7(25 \%)$ & $21(75 \%)$ & $59(69 \%)$ & $26(30 \%)$ \\
\hline Disgeusia & $11(39 \%)$ & $17(60 \%)$ & $57(67 \%)$ & $28(32 \%)$ \\
\hline $\begin{array}{l}\text { Agrandamiento } \\
\text { gingival }\end{array}$ & $4(14 \%)$ & $24(85 \%)$ & $10(11 \%)$ & $75(88 \%)$ \\
\hline
\end{tabular}

\section{DISCUSIÓN}

En un estudio publicado en el Journal of Oral and Maxillofacial Pathology en el año 2012 encontraron que un $16,99 \%$ de pacientes con medicación antihipertensiva presentaron hiposalivación, 16.9\% presentó agrandamiento gingival inducido por fármacos y un $4.5 \%$ presentó reacciones liquenoides. ${ }^{3}$

De acuerdo a un estudio de la Universidad de Talca en Chile, un 13,6\% de los pacientes con medicación antihipertensiva consideró que la cantidad de saliva en su boca era poca. ${ }^{2}$

En una publicación en el Journal of Periodontology, en el cual se estudiaron 911 pacientes para determinar la prevalencia de agrandamiento gingival inducido por los antagonistas del calcio, se concluyó que existe una prevalencia clínicamente significativa y esta fue de $6,3 \%, 1,7 \%$ y $2,2 \%$ para nifedipino, amlodipina y diltiazem respectivamente. ${ }^{4}$

Otro estudio concluyó que la prevalencia de hiperplasia gingival causada por medicamentos antagonistas de canales de calcio puede ser tan alta con un $38 \%$, siendo más prevalente con el nifedipino que con otros bloqueadores de calcio. Además se afirma que es 3,3 veces más frecuente en hombres que en mujeres. ${ }^{5}$ 
En un estudio realizado en India y publicado en el Journal of Dental and Medical Sciences por el Dr. Shantala Arunkumar 34, se encontró que de los 603 pacientes con los cuales fue realizado el estudio, 382 (67.4\%) presentaron algún tipo de manifestación oral, a diferencia del presente estudio, el cual fue realizado en 113 pacientes hipertensos y seencontró que $91(80.53 \%)$ pacientes presentaron algún tipo de manifestación oral.

Dentro de las manifestaciones orales más comúnmente encontradas en este estudio se tiene a la disgeusia con un $60 \%$, seguido de hiposalivación con un $58 \%$ y por último agrandamiento gingival, siendo la menos prevalente, con un $12 \%$. En comparación con el estudio publicado en el Journal of Dental and Medical Sciences por el Dr. Shantala Arunkumar 34, en el cual la manifestación oral máscomún fuexerostomía con un $25.5 \%$, seguido de disgeusia con un $17.7 \%$ y por último agrandamiento gingival con un $9 \%$.

En un artículo realizado en India en el año 2012 por Kumar y colaboradores, 3 y publicado en el Journal of Oral and Maxillofacial Pathology, las manifestaciones orales que se encontraron con mayor frecuencia fueron agrandamiento gingival e hiposalivación, ambas con un 17\%.

En el presente estudio se destaca una mayor prevalencia de hiposalivación en mujeres con un $69 \%$, resultados similares fueron encontrados en el artículo "Hiposalivación inducida por drogas antihipertensivas", ${ }^{24}$ publicado en el Acta Odontológica Venezonala, en el cual también se describe una mayor prevalencia de hiposalivación en mujeres con un $79 \%$.

\section{CONCLUSIONES}

Se puede concluir que un alto porcentaje de los pacientes hipertensos, a los que se le realizó este estudio, presentaron manifestaciones orales. Se encontró que existe una mayor prevalencia de hiposalivación y disgeusia en mujeres, sin embargo la hiperplasia gingival fue mas prevalenteen hombres. Tanto la hiposalivación, como disgeusia $\mathrm{y}$ agrandamiento gingival se presentaron en $\mathrm{pa}^{-}$ cientes que llevaban menos de un año de ingesta del medicamento. La disgeusia e hiperplasia gingival fueron más prevalentes en pacientes que llevaban tomando más de tres años la medicación antihipertensiva.
Sin embargo la hiposalivación fue más prevalente en pacientes que llevaban tomando menos de un año el medicamento.

\section{RECOMENDACIONES}

Se recomienda también, la realización de estudios más objetivos en cuanto a los registros de hiposalivación, ya que la sequedad bucal es una queja subjetiva y puede no estar relacionada con la disminución de la saliva en los pacientes. Por ésto es recomendable realizar estudios más profundos en los cuales se evalúe este parámetro por medio de sialometría, sialografía u otros exámenes que puedan evidenciar una verdadera hipofunción de las glándulas salivales. También es recomendable la realización de estudios más específicos en cuanto al agrandamiento gingival, en el cual se implementen métodos para evaluar la cantidad de placa bacteriana, para así poder confirmar si el agrandamiento es inducido en sí por el fármaco antihipertensivo.

\section{REFERENCIAS BIBLIOGRÁFICAS}

1. Kotchen TA. Hypertensive vascular disease. In: Longo D, Jameson J, Fauci A, Kasper D, Hauser S, Loscalzo J; editors. Harrison's principles of internal medicine. 18th ed. McGraw-Hill; 2011. p.2024-2059

2. Sánchez D. Estado de salud oral, conocimientos, actitudes, prácticas y autopercepción de pacientes hipertensos atendidos en el centro de salud familiar Dr. Carlos Díaz Gidi de la Comuna de San Javier, Región deMaule. [Abstract de tesis degrado]. Chile: Universidad de Talca, Facultad de Odontología; 2010.

3. Kumar P, Chowdhary R, Shanmugan K. Oral manifestations in hypertensive patients: A clinical study. Journal of Oral and Maxilofacial Pathology. [Revista Online] 2012; [Consultado 10 de Septiembre de 2013] (Vol. 16) [pp. 215-221] Disponibleen: http://www.ncbi. nlm.nih.gov/pmc/articles/PMC3424937/

4. Ellis JS, Seymour RA, Steele JG, Robertson P, Butler TJ, Thomason JM. Prevalence of gingival overgrowth induced by calcium channel blockers: A communitybased study. J Periodontology. [Revista Online] 1999; [Consultado en 20 de Enero de 2014] (Vol.70) [pp. 6367]. Disponible en: http://www.joponline.org/doi/ pdf/10.1902/jop.1999.70.1.63

5. Michael Prisant L, Wayne H. Calcium Channel Blocker Induced Gingival Overgrowth. The Journal of Clinical Hypertension. [Revista Online] Julio - Agosto 2002 [Consultado 29 de Septiembre de 2013] (Vol. 4, No.4). Disponible en: http://onlinelibrary.wiley.com/ doi/10.1111/j.1524-6175.2002.01095.x/abstract;jsess ionid=B55F80E3B15C50173142F42B45C32307.f02t03

6. Sánchezetal. Guías Latinoamericanas deHipertensión Arterial. Revista Chilena deCardiología. [Revista Online] 
Mar. 2010;[Consultado 20 de Enero de 2014] (Vol. 29) [pp. 117-144]. Disponible en: http://www.scielo.cl/pdf/ rchcardiol/v29n1/art12.pdf

7. Hall J.E. Visión general dela circulación; física médica de la presión, el flujo y la resistencia. En: Hall J.E., editor. Compendio defisiología médica. 11a ed. Madrid. Elsevier; 2007. p. 95-100.

8. CTO. ManualCTO: Cardiología. Madrid: CTOEditorial; 2012.

9. Pickering TG, HallJE, AppelLJ, etal. Recommendations for blood pressure measurement in humans and experimentalanimals. Part 1: blood pressure measurement in humans: a statement for professionals from the subcommittee of professional and public education of the American Heart Association Council on High Blood Pressure Research. Hypertension. 2005; 45 142-161

10. Roca R.G. Hipertensión Arterial. En: RobertZ., Hecheverría G., editores.Temas demedicina interna. 4ta ed. La Habana. Editorial Ciencias Médicas; 2002. p 325-358.

11. Gazitúa R. [Página principal en internet]. Santiago: Universidad Católica de Chile; c2007 [Actualizado 2007, Sep; consultado 7 Abril 2014]. Disponible en: http:// escuela.med.puc.cl/Publ/ManualSemiologia/210Pres ionArterial.htm

12. ArmijoJA. Mediavilla A. Fármacos antihipertensores. En: Flórez J; editor. Farmacología Humana. 3ra ed. Masson S.A.; 1998. p. 671-683.

13. August $\mathrm{P}$. Initial treatment of hypertension. N Engl J Med [Internet]. 2003 [Consultado 10 Abr 2014]; 348 (7): 610-617. Disponibleen: www.njem.org

14. Smitha K. Amlodipine-induced gingival overgrowth in a Patient with uncontrolled type 2 diabetes mellitus with hypercholesterolemia: A casereport. Clin Adv Perio. 2012; 2 (2): 115-122.

15. Finkel R., Clark MA, Cubeddu LX. Antihypertensives. In: Harvey R, ChampeP, editors. Lippincott'sillustrated reviews: pharmacology. 4th ed. Lippincott Williams \& Wilkins; 2009. p 215-228.

16. Andersen L, Kragelund C, Relbel J, Nauntofte B. Oral adverse drug reactions to cardiovascular drugs. Crit Rev Oral Biol Med [Internet]. 2004 [Consultado 5 Dic 2013]; 15 (1): 28-46. Disponible en: cro.sagepub.com

17. FoiseCK, Getka TP. Drug Induced GingivalEnlargement. Clinical Update. Naval Postgraduate Dental School. National Naval Medical Center. 2008; 30 (6): 1-2.

18. Sapp P J, Eversole L R, Wysocki G P. Lesiones del tejido conjuntivo. En: Sapp P J, Eversole L R, Wysocki G P, editors. Patologia oral y maxilofacial contemporánea. 2da ed. España. Elsevier; 2004. P 287-329.

19. Seymour RA, Thomason JM, Ellis JS. The pathogenesis of drug induced gingival overgrowth. JClin Periodontol. 1996; 23 (3): 165-175.

20. Ikawa K, Ikawa M, Shimauchi H, Iwakura M, Sakamoto S. Treatment of gingival overgrowth induced by ma- nidipine administration. A case report. J Periodontol. 2002; 73 (1): 115-122.

21. Sapp P J, Eversole L R, Wysocki G P. Lesiones físicas y químicas. En: Sapp PJ, Eversole LR, WysockiGP, editors. Patologia oral y maxilofacial contemporánea. 2da ed. España. Elsevier; 2004. P 366-392.

22. Turner MD, ShipJA. Dry mouth and its effects on theoral health of elderly people.JADA [Internet]. 2007 [Consultado 5Dic 2013]; Vol. 138: 15s-20s. Disponibleen: http://jada. ada.org

23. Scully C, Bagan-Sebastian JV. Adverse drug reactions in the orofacial region. Crit Rev Oral Biol Med. 2004; 15(4): 221-240.

24. Átilas N, Costa M, Morais R, Ribeiro A, Generoso R. Hiposalivación inducida por drogas antihipertensivas. Acta Odontológica Venezolana. [Internet]. 2009 [Consultado 13 Mar 2014]; 47(1): 1-8. Disponible en: www.actaodontologica.com/ediciones/2008/1/hiposalivacion_inducida_drogas_antihipertensivas.asp

25. Fox P. Xerostomia: Recognition and Management. Supplement to Access. [Internet]. 2008. [Consultado 17 Mar 2014]; 2-7. Disponible en: http://www.adha.org

26. Silvestre F, Miralles L, Martínez V. Tratamiento de la boca seca: puesta al día. Med Oral. 2004; 9: 273-279.

27. Gupta A, Epstein Jm Srousii H. Hyposalivation in elderly patients. JCan Dent Assoc. 2006; 72(9): 841-846

28. A betterunderstanding of drug induced tastedisturbances may improve management of the condition. Drug Ther Perspect. 2008. 24 (12): 22-24

29. Douglass R, Heckman G. Drug related tastedisturbance. Can Fam Physician. 2010. 56(11): 1142-1147.

30. Henkin RI. Drug induced taste and smell disorders. Incidence, mechanisms and managemente related primarily to treatment of sensory receptor dysfunction. [Abstract]. Drug Safety. 1994. 11(5): 318-377.

31. Sapp PJ, Eversole LR, Wysocki GP. Trastornos mediados por procesos inmunitarios. En: Sapp P J, Eversole L R, WysockiG P, editors. Patologia oral y maxilofacial contemporánea. 2da ed. España. Elsevier; 2004. P 252-286

32. Serrano-SánchezP, BagánJV, Jiménez-Soriano, Sarrión G. Drug induced ora lichenoid reactions. A literature review. JClin Exp Dent. 2010. 2(2): 71-75.

33. Backmann K, Jontell M. Microbial associated oral lichenoid reactions. Oral Dis. 2007. Vol1 13: 402-406.

34. Arunkumar S, Kalappanavar AN, Annigeri RG, Shakunthala GK. Adverse oral manifestations of cardiovascular drugs. IOSR-JDMS. [Internet]. 2013. [Consultado 5 Dic 2013]; 7 (5): 64-71. Disponible en: www.iosrjournals.org 